\title{
Author Correction: Ritualised Dung Kicking by White Rhino Males Amplifies Olfactory Signals but Reduces Odour Duration
}

\author{
C. Marneweck $^{1,2} \cdot$ A. Jürgens ${ }^{3} \cdot$ A. M. Shrader ${ }^{1,4}$ \\ Published online: 11 March 2019 \\ (C) Springer Science+Business Media, LLC, part of Springer Nature 2019
}

\section{Author Correction: Journal of Chemical Ecology (2018) 44:875-885 https://doi.org/10.1007/s10886-018-0988-3}

The original version of this article unfortunately contained some mistakes. Firstly, Fig. 4 listed a contaminant. The correct version of Fig. 4 can be found here. Second, an incorrect version of Supplementary Table S1 was mistakenly included. A corrected and updated version of Supplementary Table S1 can be found here as well, pertaining specifically to the important compounds highlighted in Fig. 4 and with additional chemical information included.

The authors apologize for this oversight and for any confusion it may have caused.

The online version of the original article can be found at https://oi.org/ 10.1007/s10886-018-0988-3

C. Marneweck

courtney.marneweck@gmail.com

1 School of Life Sciences, University of KwaZulu-Natal, Pietermaritzburg, South Africa

2 Present address: School of Biology and Environmental Sciences, University of Mpumalanga, Mbombela, South Africa

3 Chemical Plant Ecology, Technische Universität Darmstadt, Darmstadt, Germany

4 Mammal Research Institute, Department of Zoology and Entomology, University of Pretoria, Pretoria, South Africa 
a) Intact

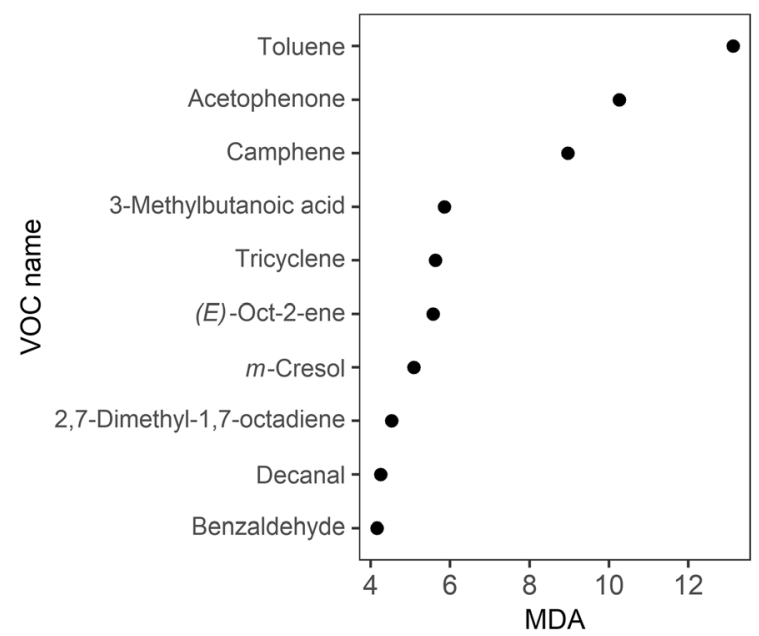

b) Dispersed

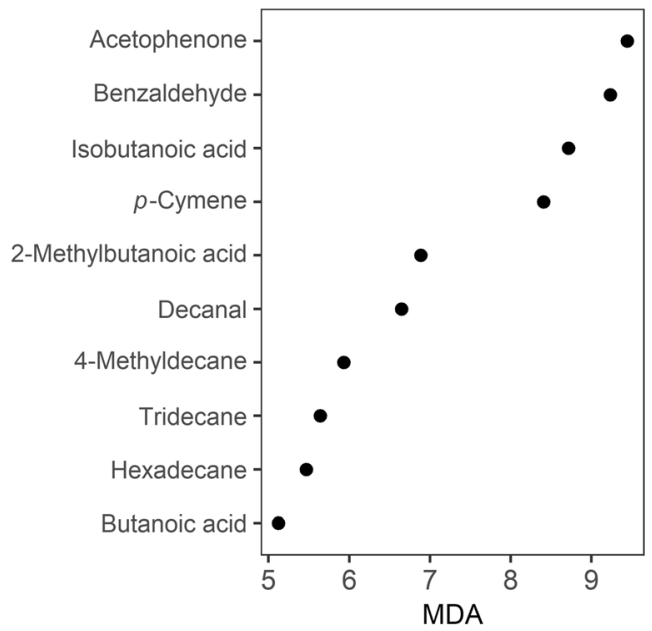

Fig. 4 The importance of volatile organic compounds (VOCs) for distinguishing the age of dung when a intact and $\mathbf{b}$ dispersed. Importance was based on mean decrease in accuracy (MDA). Only the top ten compounds are presented in the figure

Table S1 Chemical information pertaining to the tentative identification of compounds listed in Fig. 4 (the importance of volatile organic compounds for distinguishing the age of dung)

\begin{tabular}{|c|c|c|c|c|c|c|}
\hline Compound & $\begin{array}{l}\text { Retention } \\
\text { time (min) }\end{array}$ & $\begin{array}{l}\text { Retention } \\
\text { index RI (calc.) }\end{array}$ & $\begin{array}{l}\text { Retention } \\
\text { index RI (lit.) }\end{array}$ & $\begin{array}{l}\text { Observed Match and } \\
\text { R. Match values }\end{array}$ & $\begin{array}{l}\text { Lit. Source of RI index / Lit. } \\
\text { Source of MS (NIST 2013) }\end{array}$ & $\begin{array}{l}\text { MS-Spectrum in Sample } \\
\text { (Most abundant peaks) }\end{array}$ \\
\hline \multicolumn{7}{|l|}{ Alkanes } \\
\hline 4-Methyldecane ${ }^{\mathrm{b}}$ & 9.00 & 1017 & 1059 & Match: 886 R.Match: 905 & Zaikin and Borisov (2002) & \\
\hline Tridecane $^{\mathrm{c}}$ & 15.22 & 1300 & 1300 & Match: 922 R.Match: 947 & & \\
\hline Hexadecane $^{\mathrm{c}}$ & 20.64 & 1600 & 1600 & Match: 813 R.Match: 895 & & \\
\hline \multicolumn{7}{|l|}{ Alkenes } \\
\hline$(E)-2-$ Octene ${ }^{\mathrm{b}}$ & 4.32 & 799 & 798 & Match: 799 R.Match: 886 & Xu et al. (2003) & \\
\hline \multicolumn{7}{|l|}{ Aliphatic Aldehydes } \\
\hline Decanal $^{\mathrm{c}}$ & 13.17 & 1198 & 1205 & Match: 766 R.Match: 819 & Baccouri et al. (2007) & \\
\hline \multicolumn{7}{|l|}{ Aliphatic Acids } \\
\hline Isobutanoic acid $^{\mathrm{b}}$ & 3.40 & 740 & 765 & Match: 808 R.Match: 870 & Mateo and Zumalacarregui (1996) & \\
\hline Butanoic acid $^{\mathrm{b}}$ & 4.28 & 797 & 790 & Match: 697 R.Match: 765 & Quijano et al. (2007) & \\
\hline 3-Methylbutanoic acid ${ }^{\mathrm{b}}$ & 4.98 & 831 & 831 & Match: 664 R.Match: 724 & Ventanas et al. (2008) & \\
\hline 2-Methylbutanoic acid ${ }^{\mathrm{b}}$ & 5.40 & 851 & 853 & Match: 690 R.Match: 745 & Kondjoyan et al. (1997) & \\
\hline \multicolumn{7}{|c|}{ Aromatic compounds } \\
\hline Toluene $^{\mathrm{c}}$ & 3.73 & 761 & 762 & Match: 923 R.Match: 950 & Moon et al. (2006) & \\
\hline Benzaldehyde $^{c}$ & 7.77 & 960 & 948 & Match: 817 R.Match: 892 & Jordan et al. (2001) & \\
\hline$p$-Cymene ${ }^{\mathrm{b}}$ & 9.26 & 1030 & 1060 & Match 643 R.Match 683 & Marongiu et al. (2004) & \\
\hline Acetophenone ${ }^{\mathrm{c}}$ & 10.14 & 1073 & 1062 & Match: 814 R.Match: 901 & Schwambach and Peterson (2006) & \\
\hline$m$-Cresol ${ }^{\mathrm{c}}$ & 10.53 & 1093 & 1077 & Match: 925 R.Match: 934 & Baccouri et al. (2007) & \\
\hline \multicolumn{7}{|l|}{ Terpenoids } \\
\hline Tricyclene $^{\mathrm{b}}$ & 6.98 & 925 & 923 & Match: 762 R.Match: 861 & Pino et al. (2005) & \\
\hline Camphene $^{\mathrm{b}}$ & 7.62 & 954 & 945 & Match: 919 R.Match: 962 & Zhang et al. (2005) & \\
\hline Unknown terpenoid $\mathrm{A}^{\mathrm{a}}$ & 7.07 & 929 & - & - & & $\begin{aligned} 55=999|41=981| \\
67=847|39=653| \\
68=473|95=406| \\
53=272|56=262| \\
69=260|81=245|\end{aligned}$ \\
\hline
\end{tabular}

${ }^{\mathrm{a}}=$ comparison of MS with published data

${ }^{\mathrm{b}}=$ comparison of MS and retention time with published data

${ }^{\mathrm{c}}=$ comparison of MS and retention time with authentic standard 


\section{References}

Baccouri B, Ben Temime S, Campeol E, Cioni PL, Daoud D, Zarrouk M (2007) Application of solid-phase microextraction to the analysis of volatile compounds in virgin olive oils from five new cultivars. Food Chem 102:850-856

Jordan MJ, Shaw PE, Goodner KL (2001) Volatile components in aqueous essence and fresh fruit of Cucumis melo cv. Athena (muskmelon) by GC-MS and GC-O. J Agric Food Chem 49:5929-5933

Kondjoyan N, Viallon C, Berdague JL, Daridan D, Simon M-N, Legault C (1997) Analyse comparative de la fraction volatile de jambons secs de porcs Gascon et Large-White x Landrace Francais. J Rech CNRS 29:405-410

Marongiu B, Piras A, Porcedda S (2004) Comparative analysis of the oil and supercritical $\mathrm{CO} 2$ extract of Elettaria cardamomum (L.) maton. J Agric Food Chem 52:6278-6282

Mateo J, Zumalacarregui JM (1996) Volatile compounds in chorizo and their changes during ripening. Meat Sci 44:255-273

Moon S-Y, Cliff MA, Li-Chan ECY (2006) Odour-active components of simulated beef flavour analysed by solid phase microextraction and gas chromatography-mass spectrometry and -olfactometry. Food Res Int 39:294-308

Pino JA, Mesa J, Munoz Y, Marti MP, Marbot R (2005) Volatile components from mango (Mangifera indica L.) cultivars. J Agric Food Chem 53:2213-2223
Quijano CE, Salamanca G, Pino JA (2007) Aroma volatile constituents of Colombian varieties of mango (Mangifera indica L.). Flavour Fragr J 22:401-406

Schwambach SL, Peterson DG (2006) Reduction of stale flavor development in low-heat skim Milk powder via Epicatechin addition. J Agric Food Chem 54:502-508

Ventanas S, Estevez M, Andres AI, Ruiz J (2008) Analysis of volatile compounds of Iberian dry-cured loins with different intramuscular fat contents using SPME-DED. Meat Sci 79: $172-180$

$\mathrm{Xu}$ X, Stee LLP, Williams J, Beens J, Adahchour M, Vreuls RJJ, Brinkman UA, Lelieveld J (2003) Comprehensive twodimensional gas chromatography $(\mathrm{GC} * \mathrm{GC})$ measurements of volatile organic compounds in the atmosphere. Atmos Chem Phys 3: 665-682

Zaikin VG, Borisov RS (2002) Chromatographic-mass spectrometric analysis of Fishcer-Tropsch synthesis products. J Anal Chem USSR (Engl Transl) 57:544-551

Zhang F, Xu Q, Fu S, Ma X, Xiao H, Liang X (2005) Chemical constituents of the essential oil of Asarum forbesii maxim (Aristolochiaceae). Flavour Fragr J 20:318-320 